\title{
Eddy-Current Analysis of Double-Stator Inset-Type Permanent Magnet Brushless Machines
}

\author{
Shuangxia Niu, K. T. Chau, Senior Member, IEEE, Jiangui Li, Student Member, IEEE, and \\ Wenlong Li, Student Member, IEEE
}

\begin{abstract}
In this paper, a new multi-pole double-stator inset-type permanent magnet (PM) machine is proposed for low-speed direct-drive applications. In the outer stator, a fractional-slot concentrated winding is adopted to reduce the slot number and stator yoke height, hence saving the space and improving the torque density. In the inner stator, a vernier structure is used to reduce the winding slots and enlarge the slot area to accommodate more conductors, hence fully utilizing the inner stator space. Consequently, the torque density is improved, and the cogging torque is reduced. Since the machine structure is so unique while its operating principle is so distinct, a nodal method based network-field coupled time-stepping finite element method (NF-TS-FEM) is newly developed. The corresponding modeling and analysis are simpler and more convenient than its loop method based counterpart. The analysis of eddy-current loss in both of the PMs is conducted. The performance of the proposed machine is verified by the proposed NF-TS-FEM.
\end{abstract}

Index Terms-Double-stator, eddy-current loss, inset-type, NF-TS-FEM, nodal method, PM machine.

\section{INTRODUCTION}

$\mathbf{H}$ IGH-TORQUE, low-speed permanent magnet (PM) machines have a wide range of direct-drive applications, such as wind power generation and electric vehicle propulsion. For low-speed PM machine design, the use of fractional-slot concentrated windings is preferred to that of integral-slot windings [1]. Its unique feature is that each coil is wound on one tooth and the slot number per pole per phase is less than one. On the other hand, a vernier PM machine structure is particularly attractive for low-speed application since it can produce low-speed space harmonics in the airgap field with only a few armature pole-pairs and slots [2].

In this paper, the fractional-slot concentrated windings and vernier PM machine structure are ingeniously incorporated into a compact double-stator PM machine. In the outer stator with fractional-slot concentrated windings, the slot number is small and the stator yoke height is low, hence the volume is small. In the inner stator with the vernier structure, it needs only a few winding slots so that the slot area can be large and more conductors can be accommodated.

Manuscript received October 18, 2009. First published March 01, 2010; current version published May 28, 2010. This work was supported by a Grant (HKU 7105/07E) from the Research Grants Council, Hong Kong Special Administrative Region, China.

The authors are with the Department of Electrical and Electronic Engineering, The University of Hong Kong, Hong Kong (e-mail: sxniu@eee.hku.hk).

Digital Object Identifier 10.1109/TASC.2009.2039782
The eddy-current loss in the machine can be estimated by using 2-dimensional (2-D) finite-element method (FEM), but suffers from insufficient accuracy [3]. A 3-D eddy-current FEM model can be established to accurately analyze the eddy-current loss. However, the computing time is long and the pre- and postprocessing of the model is complex. Recently, a loop method based circuit-field-torque time-stepping FEM (CFT-TS-FEM) has been proposed to calculate the eddy-current loss [4]. Compared with the 3-D FEM, the corresponding computing time is greatly reduced. However, in the eddy-current region, because of the additional unknown branch current introduced into the branch equations, the modeling is still complicated. In this paper, a nodal method based network-field one (NF-TS-FEM) is newly proposed to analyze the eddy-current loss in the proposed PM machine.

The CFT-TS-FEM in [4] is based on the loop method. In the corresponding region of conductors, the unknown loop and branch currents are introduced so that the loop-to-branch incidence matrix needs to be established. On the other hand, the NF-TS-FEM in this paper is based on the nodal method. In the corresponding region of conductors, the unknown terminal and branch voltage are introduced so that the node-to-branch incidence matrix needs to be established. When the model is complex, it is not convenient to establish the loop-to-branch incidence matrix, whereas it is very easy to establish the node-tobranch incidence matrix [5]. Also, the system equation of the NF-TS-FEM is much simpler than that of the CFT-TS-FEM. Nevertheless, once their system equations are established, their calculated results for a specific problem are the same.

\section{MACHINE STRUCTURE}

The proposed double-stator inset-type PM machine has 11 pole-pairs with 24 slots in the outer stator, 1 pole-pair with 3 slots in the inner stator, and 11 pole-pair inset-type PMs in the rotor as shown in Fig. 1.

For the outer stator, the fractional-slot concentrated windings are used, and the slot number $N_{s}$ and pole number $2 p$ are related by:

$$
2 p=N_{s} \pm 2
$$

In this design, $N_{s}=24$ and $2 p=22$ are selected. This combination of slots and poles in the outer stator has the following merits [6]:

- The fractional-slot concentrated winding arrangement in the outer stator can shorten the end-windings, thus improving the utilization of copper materials, and reducing the copper losses. The machine efficiency is increased. 


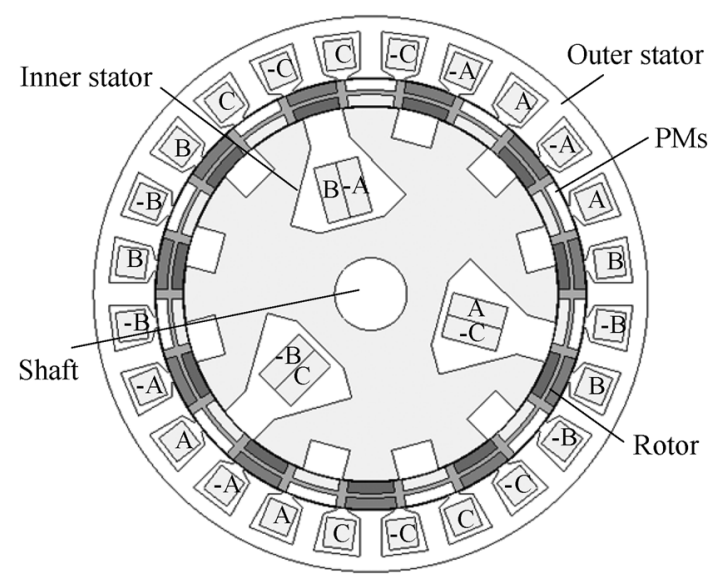

Fig. 1. Proposed machine.

- Since there are 24 slots in the outer stator and 22 poles in the rotor, the slot pitch is $11 / 12$ pole pitch. This arrangement of fractional number of slots per pole per phase can significantly reduce the cogging torque.

- The multi-pole structure leads to minimize the core yoke height, and reduces the iron used. This structure can further save the material and increase the torque density.

- Since each coil is wound on alternate stator teeth, the phase windings are isolated magnetically and physically. In this sense, the mutual inductance of phase windings becomes negligible, and improves the controllability.

For the inner stator, the vernier structure is adopted. The fundamental relationship is governed by:

$$
Z_{2}=Z_{1} \pm p
$$

where $Z_{1}$ is the number of flux-modulation poles on the stator surface or simply called stator teeth, $Z_{2}$ is the number of PM pole-pairs and $p$ is the number of winding pole-pairs. In this design, $Z_{1}=12, Z_{2}=11$ and $p=1$ are selected. This combination has the following advantages:

- The vernier machine structure can reduce the slot number and enlarge the slot area. Hence, more conductors can be accommodated into the inner stator, and the torque density can be improved.

- The concentrated winding structure in the inner stator can simplify the stator structure and effectively save the inner stator space, which can further improve the torque density.

- The modulation poles can modulate the low harmonic components, namely fundamental space harmonics, to the high harmonic components, namely 11 th space harmonics, in the airgap magnetic field with only a few armature polepairs and slots. The structure becomes simple and compact so that the manufacture cost can be reduced.

\section{EDDY-CURRENT ANALYSIS}

In order to accurately analyze the eddy-current loss in the proposed machine and avoid the complicated 3-D modeling, a nodal method based NF-TS-FEM is proposed.

\section{A. Airgap and Iron Core Domains}

Based on the Maxwell's equations, the field equations in the airgap and iron core domains are given by:

$$
\nabla \cdot(\nu \nabla A)=0
$$

where $A$ is the axial component of magnetic vector potential, and $\nu$ is the reluctivity of the material.

\section{B. Stator Winding Domain}

In the stator winding domain, the magnetic field circuit, EMF and branch equations are given by:

$$
\begin{gathered}
\nabla \cdot(\nu \nabla A)+\frac{d_{f} N_{f}}{S_{f} a p} i_{f}=0 \\
e=\frac{d_{f} N_{f} l}{S_{f} a} \iint_{\Omega} \frac{\partial A}{\partial t} \mathrm{~d} \Omega \\
\frac{d_{f} N_{f} l}{S_{f} a} \iint_{\Omega} \frac{\partial A}{\partial t} \mathrm{~d} \Omega+R_{f} i_{f}=u_{f}
\end{gathered}
$$

where $i_{f}$ is the winding current; $d_{f}$ is the polarity ( +1 or -1 ) to represent forward path or return path; $S_{f}$ is the total cross-sectional area of the region occupied by this coil group in the solution domain; $N_{f}$ is the total conductor number of this winding; $a$ is the number of parallel branches in this winding; $p$ is the symmetry multiplier which is defined as the ratio of the original full cross-sectional area to the solution area; $u_{f}$ is the voltage on the two terminals of the stator winding; $l$ is the model depth in $z$-axis direction; and $R_{f}$ is the total DC resistance of the winding which is expressed as:

$$
R_{f}=\frac{N_{f}^{2}}{a^{2} p} \frac{l}{\sigma S_{f}}
$$

In the region of the stranded windings, it yields:

$$
i_{f}=\frac{-e+u_{f}}{R_{f}}
$$

Substituting (8) into (4), the field equation becomes:

$$
\nabla \cdot(\nu \nabla A)-\frac{d_{f} a \sigma}{N_{f} l} e+\frac{d_{f} a \sigma}{N_{f} l} u_{f}=0
$$

The branch (6) can be written as:

$$
\frac{d_{f} a \sigma}{N_{f} l} \iint_{\Omega} \frac{\partial A}{\partial t} \mathrm{~d} \Omega-\frac{1}{l p R_{f}} u_{f}=-\frac{1}{l p} i_{f}
$$

Equation (5) is an additional equation to make the global coefficient matrix symmetrical, which can also be written as:

$$
\frac{d_{f} a \sigma}{N_{f} l} \iint_{\Omega} \frac{\partial A}{\partial t} \mathrm{~d} \Omega-\frac{1}{l p R_{f}} e=0
$$

\section{Solid Conductor Domain}

In the solid conductor domain, the PMs are divided into several "solid conductor bars" and the Maxwell's equations applied in these domains can be expressed as: 


$$
\nabla \cdot(\nu \nabla A)-\sigma \frac{\partial A}{\partial t}+\frac{d_{f} \sigma}{l} u_{b a r}=0
$$

where $u_{b a r}$ is the voltage difference between the two terminals of one solid conductor bar. The current density in the solid conductor bar is given by:

$$
J_{b a r}=-d_{f} \sigma \frac{\partial A}{\partial t}+\frac{\sigma}{l} u_{b a r}
$$

Integrating the current density over the cross-sectional area of the solid conductor, the current in this solid conductor bar is given by:

$$
\begin{aligned}
i_{\text {bar }} & =-\sigma \iint_{\Omega_{\text {bar }}} \frac{\partial A}{\partial t} \mathrm{~d} \Omega+\sigma \frac{S_{\text {bar }}}{l} u_{b a r} \\
& =-\sigma \iint_{\Omega_{b a r}} \frac{\partial A}{\partial t} \mathrm{~d} \Omega+\frac{1}{R_{b a r}} u_{b a r}
\end{aligned}
$$

where $S_{f}$ is the cross-sectional area of the conductor and $R_{b a r}$ is the DC resistance of one conductor bar.

If the solid conductors satisfy the periodic boundary or anti-periodic boundary condition, they can be grouped together. Hence, it yields $S_{b a r}=p S_{f} / N_{f}, i_{b a r}=i_{f} / a, u_{b a r}=a u_{f} / N_{f}$ and $R_{b a r}=a^{2} R_{f} / N_{f}$, where $p$ is the symmetry multiplier, $i_{f}$ is the total current, $a$ is the number of parallel branches, $u_{f}$ is the voltage difference between the two terminals of the solid conductor group.

Based on the above solid conductor group equations, the field equation and circuit branch equation can be obtained as:

$$
\begin{aligned}
\nabla \cdot(\nu \nabla A)-\sigma \frac{\partial A}{\partial t}+\frac{d_{f} a \sigma}{N_{f} l} u_{f} & =0 \\
\frac{d_{f} a \sigma}{N_{f} l} \iint_{\Omega} \frac{\partial A}{\partial t} \mathrm{~d} \Omega-\frac{1}{l p R_{f}} u_{f} & =-\frac{1}{l p} i_{f}
\end{aligned}
$$

\section{External Circuit Equations}

The branch equation of the external circuits can be expressed as:

$$
\left[G_{e}\right]\left\{u_{e}\right\}=\left\{i_{e}\right\}+\left\{P_{e}\right\}
$$

where $u_{e}$ and $i_{e}$ are the branch voltage and current, respectively; $G_{e}$ is the matrix of the conductance; $P_{e}$ is the column matrix associated with voltage sources, current sources as well as the solution from the previous time step for inductive and capacitive elements.

In the solid conductor domain, the key is to model the PMs by a network having $N$ bars along the circumferential direction and $M$ slices along the axial direction as shown in Fig. 2. It can be seen that the eddy-current flow can form a complete loop which enhances the accuracy to calculate the eddy-current loss. The inter-bar resistance and the end resistance are respectively given by $R_{i}=b_{P} / N \sigma l_{M} h, n=1,2, \ldots, N$, and $R_{k}=2 R_{i}$, where $l_{M}=l_{P} / M$ is the axial length of the $n^{\text {th }}$ bar of the $m^{\text {th }}$ slice, $l_{P}$ is the axial length and $b_{P}$ is the width.

The branch voltage $u_{b}$ includes the terminal voltage of solid conductor $u_{f}$ and the external branch voltage $u_{e}$. The relation-

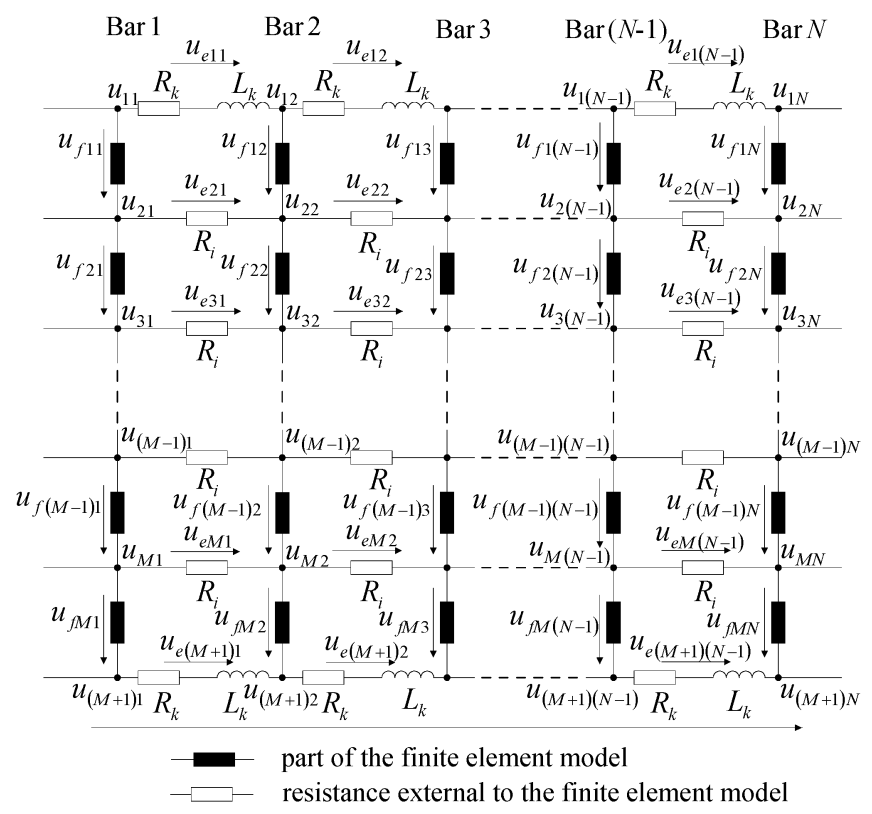

Fig. 2. Nodal method based network model.

ship between the branch voltage $u_{b}$ and the nodal voltage $u_{n}$ is given by:

$$
\begin{aligned}
& \left\{u_{b}\right\}=\left\{\begin{array}{l}
u_{f} \\
u_{e}
\end{array}\right\}=\left[\begin{array}{c}
A_{n f}^{T} \\
A_{n e}^{T}
\end{array}\right]\left\{u_{n}\right\} \\
& {\left[A_{n b}\right]=\left\{\begin{array}{l}
A_{n f} \\
A_{n e}
\end{array}\right\}}
\end{aligned}
$$

Also, the Kirchhoff's current law can be expressed as:

$$
\left[A_{n b}\right]\left\{i_{b}\right\}=0
$$

Using the Galerkin method, the coupled field and circuit equations in the magnetic field region can be written in the block matrix format. By using the back Euler's method and based on the above equations, the system equations are obtained as:

$$
\begin{aligned}
& {\left[\begin{array}{cc}
\left(\begin{array}{cc}
C_{11}+\frac{D_{11}}{\Delta t} & C_{12} \\
C_{12}^{T} & \Delta t C_{22}
\end{array}\right) & \left(\begin{array}{cc}
C_{13} & 0 \\
0 & 0
\end{array}\right) A_{n b}^{T} \\
A_{n b}\left(\begin{array}{cc}
C_{13}^{T} & 0 \\
0 & 0
\end{array}\right) & A_{n b}\left(\begin{array}{cc}
\Delta t C_{33} & 0 \\
0 & -\frac{\Delta t}{l p} G_{e}
\end{array}\right) A_{n b}^{T}
\end{array}\right] \times} \\
& \left\{\left(\begin{array}{c}
A^{k} \\
e^{k}
\end{array}\right)\right\}=\left\{\begin{array}{c}
\left(\begin{array}{c}
P_{A}+\frac{D_{11}}{\Delta t} A^{k-1} \\
C_{12}^{T} A^{k-1}
\end{array}\right) \\
u_{n b}\left(\begin{array}{c}
C_{13}^{T} A^{k-1} \\
-\frac{\Delta t}{l p} P_{e}
\end{array}\right)
\end{array}\right\}
\end{aligned}
$$

where the coefficient matrix is symmetrical. Consequently, the system equations are solved at each time step.

It should be noted that the edge effect of the 3-D machine model has been taken into account. Namely, the fringing effect is manipulated by using the effective axial length. Also, the end-winding inductance is incorporated into the circuit equation which is coupled with the FEM equation.

\section{Performance Analysis}

By using the proposed NF-TS-FEM, both the steady-state and transient performances are analyzed. Fig. 3 shows a typical mag- 


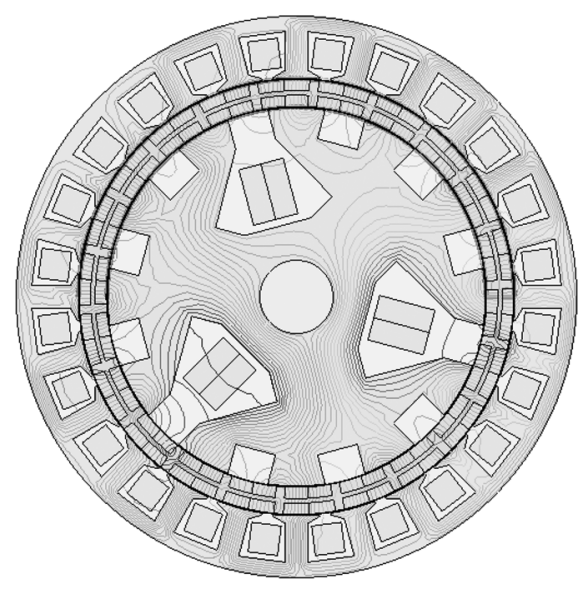

Fig. 3. Magnetic field distribution at full load.

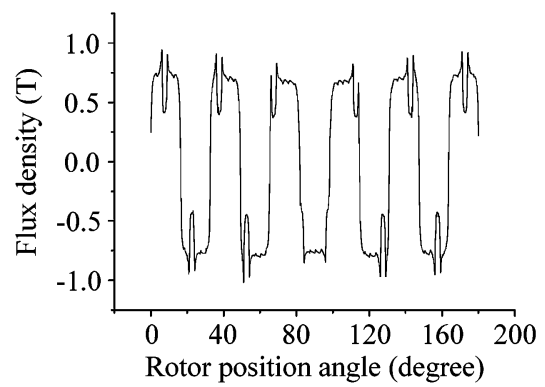

(a)

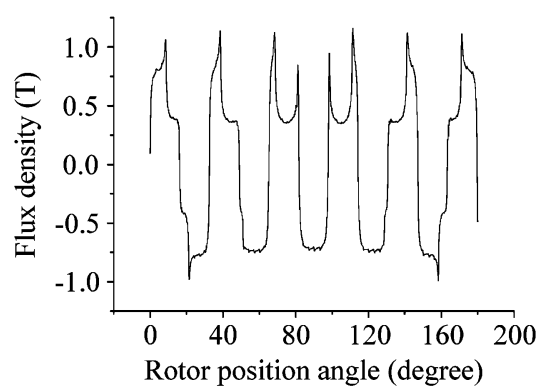

(b)

Fig. 4. Airgap flux density waveforms. (a) Outer airgap. (b) Inner airgap.

netic field distribution at full load. The corresponding flux density waveforms in the inner and outer airgaps are shown in Fig. 4. It can be found that the armature field excited by the inner stator winding is effectively modulated by the split poles on the stator teeth. Then, the transient torque response at full load is shown in Fig. 5. It confirms that the steady-state torque can achieve 115 $\mathrm{Nm}$ at the rated speed of $273 \mathrm{rpm}$. Hence, the torque density can reach $50 \mathrm{kNm} / \mathrm{m}^{3}$. Consequently, the cogging torque is shown in Fig. 6, which is only $2 \%$ of the rated torque, thus verifying that the cogging torque of the proposed machine is negligible.

It can be observed that the torque ripple is significantly larger than the cogging torque. It is due to the fact that the harmonic torque, which is mainly caused by phase commutation during brushless DC operation, dominates the torque ripple. Meanwhile, the cogging torque is very small, which is actually governed by the inverse of the smallest common multiple of the numbers of slots and pole pairs.

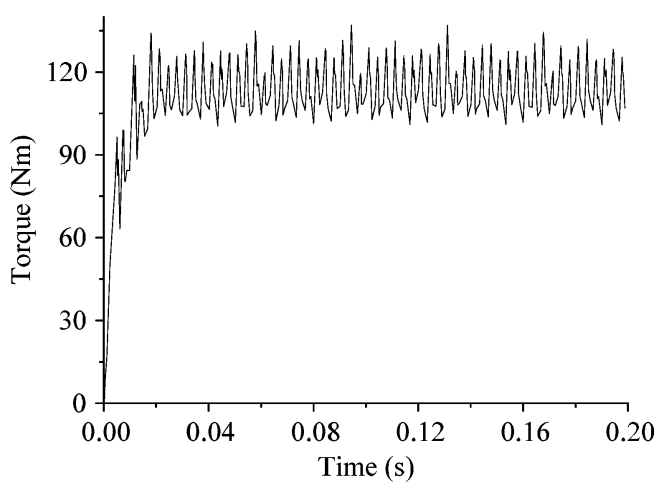

Fig. 5. Transient torque response at full load.

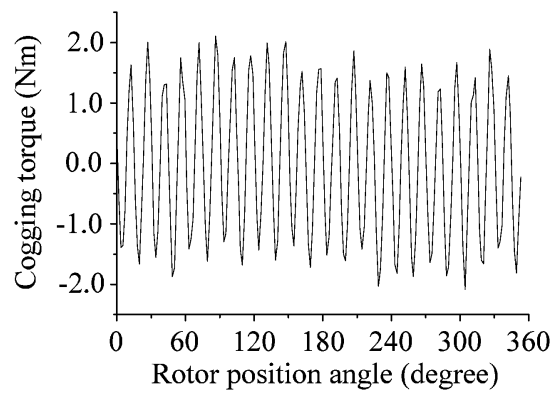

Fig. 6. Cogging torque waveform.

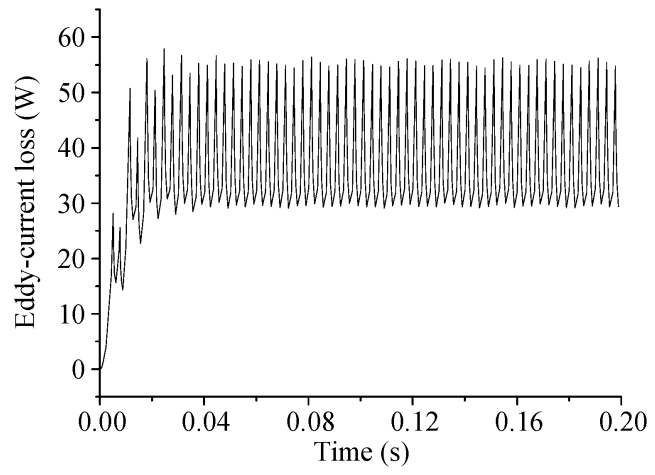

Fig. 7. Transient eddy-current loss response in PMs during commutation.

Finally, the transient response of eddy-current loss in the PMs during the commutation period is analyzed as shown in Fig. 7. It can be observed that the eddy-current loss increases drastically within the commutation time, which is actually due to the fact that there is a sudden large change of armature current field during commutation.

The eddy current loss analysis is calculated under the rated condition of $115 \mathrm{Nm}$ and $273 \mathrm{rpm}$ with the time step of $0.1 \mathrm{~ms}$. The iron core is based on M19_24G silicon steel sheets. The $\mathrm{PM}$ material is sintered $\mathrm{NdFeB}$ with the remanent flux density of $1.3 \mathrm{~T}$ and the coercive force of $940 \mathrm{kA} / \mathrm{m}$.

\section{CONCLUSION}

In this paper, a new multi-pole double-stator inset-type PM brushless machine has been proposed for low-speed direct-drive 
applications, which can offer high torque density and low cogging torque. A novel nodal method based NF-TS-FEM has been developed to analyze both the steady-state and transient performances of this machine. Particularly, the eddy-current loss in PMs of the proposed machine has been successfully modeled and calculated.

\section{REFERENCES}

[1] S. Niu, K. T. Chau, and C. Yu, "Quantitative comparison of doublestator and traditional permanent magnet brushless machines," Journal of Applied Physics, vol. 105, no. 7, pp. 07F105-07F105-3, 2009.

[2] A. Toba and T. A. Lipo, "Novel dual-excitation permanent magnet vernier machine," in IEEE IAS Annual Meeting, 1999, pp. 2539-2544.
[3] W. N. Fu and Z. J. Liu, "Estimation of eddy-current loss in permanent magnets of electric motors using network-field coupled multislice timestepping finite-element method," IEEE Trans. Magnetics, vol. 38, no. 2, pp. 1225-1228, 2002.

[4] S. Niu, K. T. Chau, and J. Z. Jiang, "Analysis of eddy-current loss in a double-stator cup-rotor PM machine," IEEE Trans. Magnetics, vol. 44, no. 11, pp. 4401-4404, 2008.

[5] W. N. Fu, P. Zhou, D. Lin, S. Stanton, and Z. J. Cendes, "Modeling of solid conductors in 2-D transient finite element analysis and its application to electric machines," IEEE International Electric Machines and Drives Conference, pp. 1272-1278, 2003.

[6] S. Niu, K. T. Chau, J. Z. Jiang, and C. Liu, "Design and control of a new double-stator cup-rotor permanent-magnet machine for wind power generation," IEEE Trans. Magnetics, vol. 43, no. 6, pp. 2501-2503, 2007. 